\title{
Dynamic response of shear-flexible cylindrical isotropic shells with clamped edges
}

\author{
Zafer I. Sakka ${ }^{\text {, Jamal A. Abdalla }}{ }^{\mathrm{b}, *}$ and H.R.H. Kabir ${ }^{\mathrm{c}}$ \\ ${ }^{a}$ University of New South Wales, Australia \\ ${ }^{\mathrm{b}}$ Department of Civil Engineering, American University of Sharjah, UAE \\ ${ }^{\mathrm{c}}$ Department of Civil Engineering, Kuwait University, Kuwait
}

Received 20 June 2005

Revised 23 September 2005

\begin{abstract}
It is fundamental to obtain the natural frequencies and the corresponding mode shapes for cylindrical shells in order to determine their response to different dynamic loading. In this paper an analytical investigation to the free vibration response of moderately-thick shear flexible isotropic cylindrical shells with all edges clamped is presented. The Sander's kinematic relations for moderately thick cylindrical shell panels are utilized to develop the governing partial differential equations in conjunction with the boundary conditions. A recently developed generalized Navier's approach, based on a boundary continuous double Fourier series expansion, is used as a solution methodology. A parametric study is presented with respect to various thicknesses, length and radius of curvature of the shell panel. The convergence of the solution method is established numerically for various parametric properties. The present results are compared with the results obtained from finite element method using a four-node isoparametric shell element. The results thus presented should serve as bench-mark solutions for future comparisons with numerical and approximate methods for calculation of free vibration parameters of moderately-thick isotropic cylindrical shells.
\end{abstract}

Keywords: Vibration analysis, cylindrical isotropic shell, frequency response, shear-flexible

\section{Introduction}

Shells have been recognized and used in construction of monumental and landmark structures since the dawn of civilization. Various shell forms such as spherical dome shells, cylindrical shells and other curved surfaces are routinely used in civil, mechanical and marine structures. Shells or curved surfaces have become more popular lately in modern advanced industries, such as aerospace, aircraft, hydrospace, automobile, oil and LNG tanks, pipelines, tunnels and other land based applications. These industries are continuously experiencing development of new materials that are regularly replacing the conventional materials. The advent of these materials is due to their unique and favorable properties such as high strength-to-weight ratio, high stiffness-to-weight ratio, fatigue strength and high performance in maintaining structural integrity at extreme loads and temperatures. These new materials have low shear rigidities in comparison to the conventional ones. The low rigidity response coupled with the curvature effects in shell formulation brings complications in developing analytical solutions.

Over the years, static and dynamic equilibrium equations that govern the behavior of cylindrical shells have been developed by several researchers including Love [1], Donnell [2], Sanders [3], Timoshenko [4] and Fl ügge [5]. As summarized and investigated in details by Leissa [6], there are several well-known shell theories, with various

${ }^{*}$ Corresponding author. Tel.: +971 6 5152959; Fax: +971 6 5152979; E-mail:jabdalla@aus.edu. 
assumptions, that include Love - Timoshenko, Reissner - Naghdi - Berry, Sanders, Goldenveizer - Novozhilov, Flugge - Lur'ye-Byrne, Vlasov and Donnell - Mushtari, to name a few. Updated review and bibliography of recently developed shell theories and solution methodologies is given by Noor [7], Liew [8], Qatu [9] and Soedel [10]. Many approximate and exact closed-form solution methods for governing dynamic equilibrium equations have been proposed in the literature to study and predict the free vibration characteristics - frequencies and mode shapes - of cylindrical shells. As the thickness of the shell increases, first-order shear deformations as well as second-order shear deformations have to be taken into consideration. In addition, there is coupling between longitudinal, radial and circumferential displacements in the dynamic equilibrium equations of cylindrical shells that need to be considered. It is clear that closed-form solution for shells, with all these conditions taken into consideration, is a formidable task. Therefore, several assumptions have been introduced in the literature to simplify the formulation in order to come up with closed-form solutions. Similar assumptions were also made in approximate methods such as the finite element method and large number of shell finite elements was developed by several researchers. Recent advances on development of shell finite elements are surveyed by Yang et al. [11].

Wang and Lia [12] introduced a wave approach based on Love's equations to predict the natural frequencies of finite length circular cylindrical shells with different boundary conditions. Callahan and Baruh [13] introduced a systematic procedure for obtaining a closed-form eigen solution for the equations of motion of thin circular cylindrical shells and the results they obtained are very comparable to that of experimental results. Xiang et al. [14] provided exact solution for vibration of thin circular cylindrical shells with intermediate ring support based on thin elastic shell theory. In their proposed method, the state-space technique was employed to derive the homogenous differential equations system for a shell segment and a domain decomposition approach was developed to cater for the continuity requirements between shell segments. Stanley and Ganesan [15] presented a semi-analytical finite element solution for vibration of stiffened cylindrical short and long shells with clamped-clamped boundary condition. Chen et al. [16] and Chen and Ding [17] studied the coupled free vibration of transversely isotropic cylindrical shells based on three dimensional elasticity. Bessel functions solution, which includes complex arguments, are used for the case of complex eigenvalues. Ng et al. [18] used an extension of Donnell's shell theory with first-order shear deformation to investigate the dynamic stability of simply-supported, isotropic cylindrical panels under combined static and dynamic axial forces. A system of equations were generated via normal-mode expansion and the response is obtained. Kurpa et al. [19] proposed an algorithm based on the R-function theory and variational Ritz method for the free vibration of arbitrary plan-form shallow shell with different types of curvatures. Reasonable agreement was observed between the results obtained and those available in the literature. Jansen [20] and Ambili [21] tackled the nonlinear vibrations of cylindrical shells.

The shell formulation considered in this paper is based on Sander's kinematics relations, where the effect of shear deformations and rotational moment of inertia are accounted for. The inclusion of shear deformation into the shell formulation accurately predicts the shell behavior for a moderately thick situation as presented by Bert and Kumar [22] and Reddy [23]. Vibrations of thick cylindrical shells are also addressed by Vanderpool and Bert [24] and Liew, Lim and Kitipornchai [25]. A recently developed analytical solution by Chaudhuri and Kabir [26] and Kabir and Chaudhuri [27] based on boundary continuous double Fourier series expansion is utilized. Therefore, the main objective of the present work is to obtain an exact solution for free vibration response of a moderatelythick cylindrical shell panel with all edges clamped using conventional isotropic materials with low shear rigidities. Analytical solutions of such shells are absent in the literature.

Results from three finite element programs are compared with those of the analytical solution results. It is evident that the results of this analytical solution can serve as bench-mark solutions for future comparisons with numerical and approximate methods for calculation of free vibration parameters of moderately-thick isotropic cylindrical shells.

\section{Dynamic equilibrium equations}

A typical cylindrical panel of thickness $h$ and radius $R$ and length $\mathrm{b}$ is shown in Fig. 1. Reference axes $\left(x_{1}, x_{2}, x_{3}\right)$ are placed at middle surface $(h / 2)$ of the shell thickness. Axes $x_{1}, x_{2}$ and $x_{3}$ represent an orthogonal curvilinear coordinate system along circumferential (tangential), longitudinal (axial) and radial directions of the 


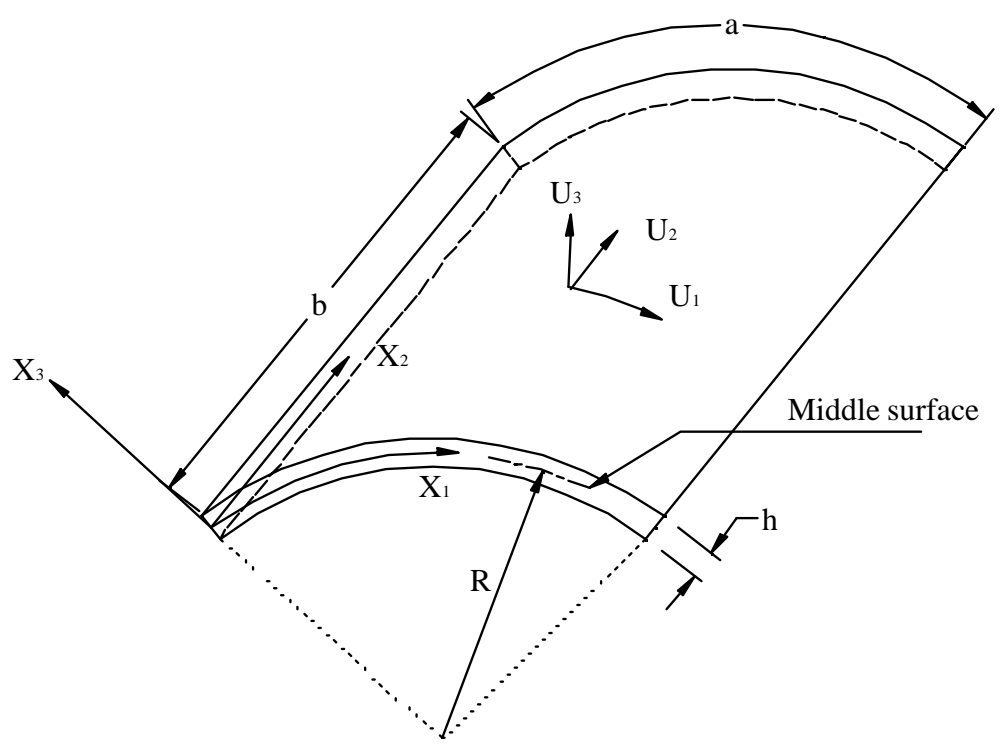

Fig. 1. A cylindrical shell panel showing dimensions, coordinate system and displacements.

shell, respectively. Axis $x_{3}$ is perpendicular to the surface of the panel, $a$ represent the circumferential length along $x_{1}$ axis and $b$ represent the longitudinal length along $x_{2}$ axis.

The displacement fields expressed in terms of reference surface (middle surface) displacements are:

$$
\begin{aligned}
& u_{1}=\left(1+\frac{x_{3}}{R}\right) \overline{\mathbf{u}}_{1}+x_{3} \phi_{1} \\
& u_{2}=\overline{\mathbf{u}}_{2}+x_{3} \phi_{2} \\
& u_{3}=\overline{\mathbf{u}}_{3}
\end{aligned}
$$

where $u_{i}(i=1,2,3)$ are the displacements of a point $x_{i}(i=1,2,3), \bar{u}_{i}$ denotes the displacements of the corresponding point at the reference surface, and $\phi_{1} \& \phi_{2}$ are the rotations of the normal to the reference surface about $x_{2}$ and $x_{1}$ coordinate axes, respectively.

The strain-displacement relations (kinematic relations) based on Sander's [3] shell theory are:

$$
\begin{aligned}
& \varepsilon_{1}=\varepsilon_{1}^{0}+x_{3} \kappa_{1} \\
& \varepsilon_{2}=\varepsilon_{2}^{0}+x_{3} \kappa_{2} \\
& \varepsilon_{4}=\varepsilon_{4}^{0} \\
& \varepsilon_{5}=\varepsilon_{5}^{0} \\
& \varepsilon_{6}=\varepsilon_{6}^{0}+x_{3} \kappa_{6}
\end{aligned}
$$

where $\varepsilon_{i}$ and $\varepsilon_{i}^{0}$ for $i=1,2,6$ at coordinates $\left(x_{1}, x_{2}, x_{3}\right)$ and $\left(x_{1}, x_{2}, 0\right)$ represent the surface parallel strain, the normal strain and the shearing strain components, respectively. While $\varepsilon_{4}$ and $\varepsilon_{5}$ are the transverse shear strain components along the $x_{2}-x_{3}$ and $x_{1}-x_{3}$ planes, respectively; and $\kappa_{i}$ for $i=1,2,6$ represent the changes in the curvature and the twist at reference surface. The parameters $\varepsilon_{i}^{0}$ and $\kappa_{i}$ are defined as follows:

$$
\begin{aligned}
& \varepsilon_{1}^{0}=\frac{\partial u_{1}}{\partial x_{1}}+\frac{u_{3}}{R} \\
& \varepsilon_{2}^{0}=\frac{\partial u_{2}}{\partial x_{2}}
\end{aligned}
$$




$$
\begin{aligned}
& \varepsilon_{4}^{0}=\frac{\partial u_{3}}{\partial x_{2}}+\phi_{2} \\
& \varepsilon_{5}^{0}=\frac{\partial u_{3}}{\partial x_{1}}+\phi_{1}-\frac{u_{1}}{R} \\
& \varepsilon_{6}^{0}=\frac{\partial u_{2}}{\partial x_{1}}+\frac{\partial u_{1}}{\partial x_{2}} \\
& \kappa_{1}=\frac{\partial \phi_{1}}{\partial x_{1}} \\
& \kappa_{2}=\frac{\partial \phi_{2}}{\partial x_{2}} \\
& \kappa_{6}=\frac{\partial \phi_{2}}{\partial x_{1}}+\frac{\partial \phi_{1}}{\partial x_{2}}-C_{o}\left(\frac{\partial u_{2}}{\partial x_{1}}-\frac{\partial u_{1}}{\partial x_{2}}\right)
\end{aligned}
$$

where $C_{o}$ is defined as $C_{o}=\frac{1}{2 R}$

The dynamic equilibrium equations for a cylindrical panel are:

$$
\begin{aligned}
& \frac{\partial N_{1}}{\partial x_{1}}+\frac{\partial N_{6}}{\partial x_{2}}+C_{o} \frac{\partial M_{6}}{\partial x_{2}}+\frac{Q_{1}}{R}=C_{1} \\
& \frac{\partial N_{6}}{\partial x_{1}}+\frac{\partial N_{2}}{\partial x_{2}}-C_{o} \frac{\partial M_{6}}{\partial x_{1}}=C_{2} \\
& \frac{\partial Q_{1}}{\partial x_{1}}+\frac{\partial Q_{2}}{\partial x_{2}}-\frac{N_{1}}{R}=C_{3} \\
& \frac{\partial M_{1}}{\partial x_{1}}+\frac{\partial M_{6}}{\partial x_{2}}-Q_{1}=C_{4} \\
& \frac{\partial M_{6}}{\partial x_{1}}+\frac{\partial M_{2}}{\partial x_{2}}-Q_{2}=C_{5}
\end{aligned}
$$

Where $N_{i}(i=1,2,6)$ are the in-plane (axial) stress resultants, $M_{i}(i=1,2,6)$ are the bending stress resultants, and $Q_{i}(i=1,2)$ are the transverse shear stress resultants. $C_{i}(i=1,2,3,4,5)$ are defined as follows:

$$
\begin{aligned}
& C_{1}=\left(P_{1}+\frac{2 P_{2}}{R}\right) \frac{\partial^{2} u_{1}}{\partial t^{2}}+\left(P_{2}+\frac{P_{3}}{R}\right) \frac{\partial^{2} \phi_{1}}{\partial t^{2}} \\
& C_{2}=P_{1} \frac{\partial^{2} u_{2}}{\partial t^{2}}+P_{2} \frac{\partial^{2} \phi_{2}}{\partial t^{2}} \\
& C_{3}=P_{1} \frac{\partial^{2} u_{3}}{\partial t^{2}} \\
& C_{4}=\left(P_{2}+\frac{P_{3}}{R}\right) \frac{\partial^{2} u_{1}}{\partial t^{2}}+P_{3} \frac{\partial^{2} \phi_{1}}{\partial t^{2}} \\
& C_{5}=P_{2} \frac{\partial^{2} u_{2}}{\partial t^{2}}+P_{3} \frac{\partial^{2} \phi_{2}}{\partial t^{2}}
\end{aligned}
$$

where 


$$
\begin{aligned}
& P_{1}=\int_{0}^{h} \rho d x_{3} \\
& P_{2}=\int_{0}^{h} \rho x_{3} d x_{3} \\
& P_{3}=\int_{0}^{h} \rho x_{3}^{2} d x_{3}
\end{aligned}
$$

where $\rho$ is the density of the material.

The in-plane stress, bending stress and shear stress resultants can be expressed in terms of the displacements and their derivatives as follows:

$$
\begin{aligned}
& N_{1}=A\left(\frac{\partial u_{1}}{\partial x_{1}}+\frac{u_{3}}{R}\right)+\nu A\left(\frac{\partial u_{2}}{\partial x_{2}}\right) \\
& N_{2}=\nu A\left(\frac{\partial u_{1}}{\partial x_{1}}+\frac{u_{3}}{R}\right)+A\left(\frac{\partial u_{2}}{\partial x_{2}}\right) \\
& N_{6}=\frac{1+\nu}{2} A\left(\frac{\partial u_{1}}{\partial x_{2}}+\frac{\partial u_{2}}{\partial x_{1}}\right) \\
& M_{1}=D \frac{\partial \phi_{1}}{\partial x_{1}}+\nu D \frac{\partial \phi_{2}}{\partial x_{2}} \\
& M_{2}=\nu D \frac{\partial \phi_{1}}{\partial x_{1}}+D \frac{\partial \phi_{2}}{\partial x_{2}} \\
& M_{6}=\frac{G h^{3}}{12}\left(\frac{\partial \phi_{2}}{\partial x_{1}}+\frac{\partial \phi_{1}}{\partial x_{2}}-C_{o}\left(\frac{\partial u_{2}}{\partial x_{1}}-\frac{\partial u_{1}}{\partial x_{2}}\right)\right) \\
& Q_{1}=k_{1}^{2} \frac{G h}{2}\left(\frac{\partial u_{3}}{\partial x_{1}}+\phi_{1}-\frac{u_{1}}{R}\right) \\
& Q_{2}=k_{1}^{2} \frac{G h}{2}\left(\frac{\partial u_{3}}{\partial x_{2}}+\phi_{2}\right)
\end{aligned}
$$

where $\nu$ is the Poisson's ratio, $k_{1}^{2}$ is the shear correction factor (usually taken as 5/6), $E$ is Young's modulus, and $A, D$ and $G$ are extensional, bending and shear rigidities, respectively. $A, D$ and $G$ are expressed in the following form:

$$
A=\frac{E h}{1+\nu^{2}} ; D=\frac{E h^{3}}{12\left(1-\nu^{2}\right)} \text { and } G=\frac{E}{2(1+\nu)}
$$

The substitution of Eqs (2a-e), (3a-h) and (7a-h) into Eqs (4a-e) yields the following five highly coupled second-order partial differential equations with constant coefficients:

$$
\begin{gathered}
\left(\frac{1+\nu}{2} A+C_{o}^{2} \frac{G h^{3}}{12}\right) \frac{\partial^{2} u_{1}}{\partial x_{2}^{2}}+\left(\nu A+\frac{1+\nu}{2} A-C_{o}^{2} \frac{G h^{3}}{12}\right) \frac{\partial^{2} u_{2}}{\partial x_{1} \partial x_{2}}+\left(\frac{A}{R_{1}}+k_{1}^{2} \frac{G h}{2 R}\right) \frac{\partial u_{3}}{\partial x_{1}} \\
+k_{1}^{2} \frac{G h}{2 R} \phi_{1}+C_{o} \frac{G h^{3}}{12}\left(\frac{\partial^{2} \phi_{1}}{\partial x_{2}^{2}}+\frac{\partial^{2} \phi_{1}}{\partial x_{1} \partial x_{2}}\right)+A \frac{\partial^{2} u_{1}}{\partial x_{1}^{2}}-k_{1}^{2} \frac{G h}{2 R^{2}} u_{1}=C_{1}
\end{gathered}
$$




$$
\begin{aligned}
& \left(\frac{1+\nu}{2} A-C_{o}^{2} \frac{G h^{3}}{12}+\nu A\right) \frac{\partial^{2} u_{1}}{\partial x_{1} \partial x_{2}}+\left(\frac{1+\nu}{2} A+C_{o}^{2} \frac{G h^{2}}{12}\right) \frac{\partial u_{2}}{\partial x_{1}}+A \frac{\partial^{2} u_{2}}{\partial x_{2}^{2}} \\
& \quad+\left(\frac{\nu A}{R}\right) \frac{\partial u_{3}}{\partial x_{2}}-C_{o} \frac{G h^{3}}{12}\left(\frac{\partial^{2} \phi_{1}}{\partial x_{1} \partial x_{2}}+\frac{\partial^{2} \phi_{1}}{\partial x_{1}^{2}}\right)=C_{2} \\
& \left(-k_{1}^{2} \frac{G h}{2 R}-\frac{\nu A}{R}-\frac{A}{R}\right) \frac{\partial u_{1}}{\partial x_{1}}-\frac{\nu A}{R_{1}} \frac{\partial u_{2}}{\partial x_{2}}-\left(\frac{A}{R^{2}}\right) u_{3}+\left(k_{1}^{2} \frac{G h}{2}\right) \\
& \quad\left(\frac{\partial^{2} u_{3}}{\partial x_{1}^{2}}+\frac{\partial^{2} u_{3}}{\partial x_{2}^{2}}+\frac{\partial \phi_{1}}{\partial x_{1}}+\frac{\partial \phi_{2}}{\partial x_{2}}\right)=C_{3} \\
& k_{1}^{2} \frac{G h}{2 R} u_{1}+C_{o} \frac{G h^{3}}{12}\left(\frac{\partial^{2} u_{1}}{\partial x_{2}^{2}}-\frac{\partial^{2} u_{2}}{\partial x_{1} \partial x_{2}}\right)-k_{1}^{2} \frac{G h}{2}\left(\frac{\partial u_{3}}{\partial x_{1}}+\phi_{1}\right)+D \frac{\partial^{2} \phi_{1}}{\partial x_{1}^{2}}+\frac{G h^{3}}{12} \frac{\partial^{2} \phi_{1}}{\partial x_{2}^{2}} \\
& \quad+\left(\nu D+\frac{G h^{3}}{12}\right) \frac{\partial^{2} \phi_{2}}{\partial x_{1} \partial x_{2}}=C_{4} \\
& C_{o} \frac{G h^{3}}{12}\left(\frac{\partial^{2} u_{1}}{\partial x_{1} \partial x_{2}}-\frac{\partial^{2} u_{2}}{\partial x_{1}^{2}}\right)-k_{1}^{2} \frac{G h}{2}\left(\frac{\partial u_{3}}{\partial x_{2}}+\phi_{2}\right)+\left(\frac{G h^{3}}{12}+\nu D\right) \frac{\partial^{2} \phi_{1}}{\partial x_{1} \partial x_{2}}+D \frac{\partial^{2} \phi_{2}}{\partial x_{2}^{2}}=C_{5}
\end{aligned}
$$

The boundary conditions all-edges clamped panel are:

$$
u_{1}=u_{2}=u_{3}=\phi_{1}=\phi_{2}=0 \text { at all edges }
$$

\section{Solution method}

The geometric boundary conditions Eq. (9) for a cylindrical panel are exactly satisfied by the following set of displacement functions as shown in Kabir [28], Chaudhuri and Kabir [26,29,30]:

$$
\begin{aligned}
& u_{1}=\sum_{m=1}^{\infty} \sum_{n=1}^{\infty} U_{m n} \sin \left(\alpha_{m} x_{1}\right) \sin \left(\beta_{n} x_{2}\right) e^{i \omega t} \\
& u_{2}=\sum_{m=1}^{\infty} \sum_{n=1}^{\infty} V_{m n} \sin \left(\alpha_{m} x_{1}\right) \sin \left(\beta_{n} x_{2}\right) e^{i \omega t} \\
& u_{3}=\sum_{m=1}^{\infty} \sum_{n=1}^{\infty} W_{m n} \sin \left(\alpha_{m} x_{1}\right) \sin \left(\beta_{n} x_{2}\right) e^{i \omega t} \\
& \phi_{1}=\sum_{m=1}^{\infty} \sum_{n=1}^{\infty} X_{m n} \sin \left(\alpha_{m} x_{1}\right) \sin \left(\beta_{n} x_{2}\right) e^{i \omega t} \\
& \phi_{2}=\sum_{m=1}^{\infty} \sum_{n=1}^{\infty} Y_{m n} \sin \left(\alpha_{m} x_{1}\right) \sin \left(\beta_{n} x_{2}\right) e^{i \omega t}
\end{aligned}
$$

where $\alpha_{m}=m \pi / a$ and $\beta_{n}=n \pi / b$.

The substitution of Eqs (10a-e) and there derivatives into Eqs (8a-e) will yield $15 \times m \times n$ algebraic equations with $5 \times m \times n$ unknowns, thus failing to provide a unique solution to this physical problem. Therefore, since the displacement functions and their derivatives before their substitution into the governing equations satisfy the boundary conditions without inviting any kind of discontinuities, the following series expansion of $\cos \left(\alpha_{m} x_{1}\right) \cos \left(\beta_{n} x_{2}\right)$, $\sin \left(\alpha_{m} x_{1}\right) \cos \left(\beta_{n} x_{2}\right)$ and $\cos \left(\alpha_{m} x_{1}\right) \sin \left(\beta_{n} x_{2}\right)$ are suggested for the displacement derivatives as shown in 

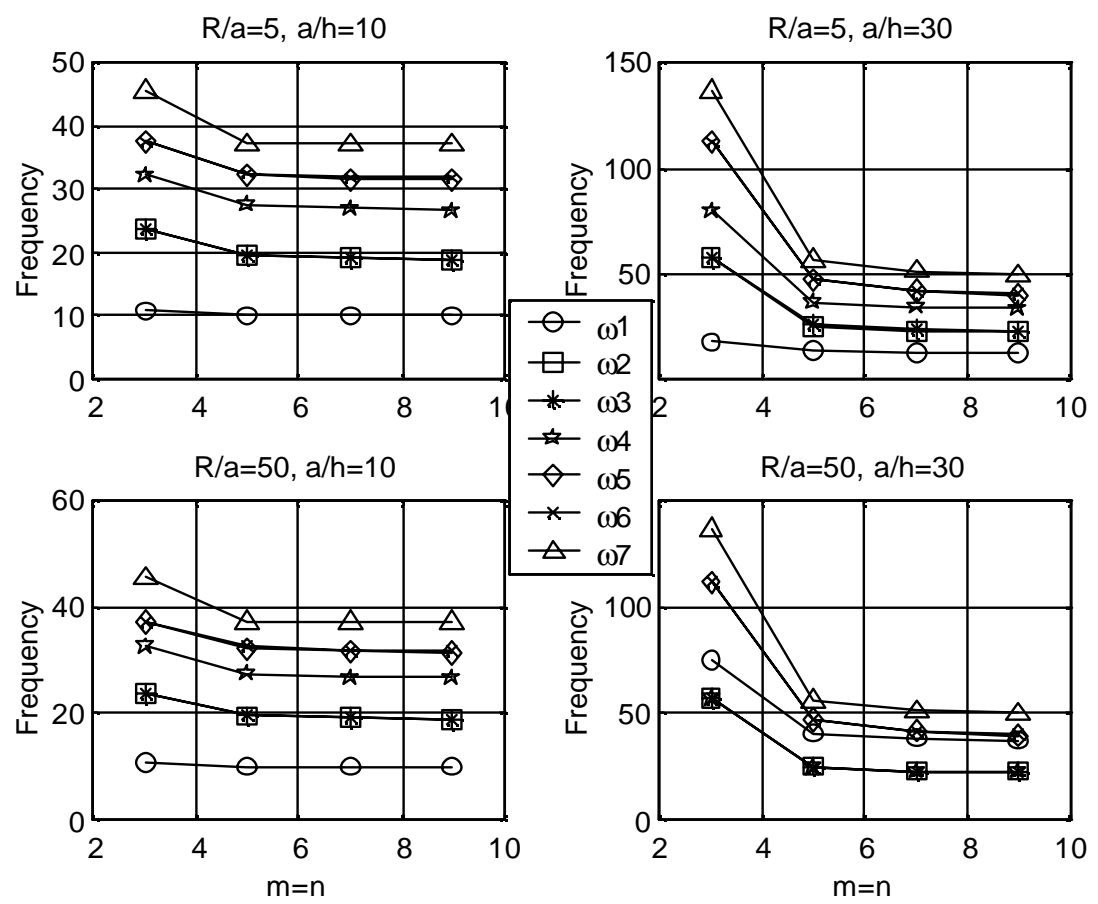

Fig. 2. Normalized natural frequencies for isotropic cylindrical panel with $b / a=1$.

Green [31], Kabir and Chaudhuri [27], Kabir [28] and Chaudhuri and Kabir [26,29,30].

$$
\begin{aligned}
& \cos \left(\alpha_{m} x_{1}\right) \sin \left(\beta_{n} x_{2}\right)=\sum_{\bar{m}}^{\infty} f_{\bar{m} m} \sin \left(\gamma_{\bar{m}} x_{1}\right) \sin \left(\beta_{n} x_{2}\right) \\
& \sin \left(\alpha_{m} x_{1}\right) \cos \left(\beta_{n} x_{2}\right)=\sum_{\bar{n}}^{\infty} g_{\bar{n} n} \sin \left(\alpha_{m} x_{1}\right) \sin \left(\psi_{\bar{n}} x_{2}\right) \\
& \cos \left(\alpha_{m} x_{1}\right) \cos \left(\beta_{n} x_{2}\right)=\sum_{\bar{n}}^{\infty} \sum_{\bar{m}}^{\infty} f_{\bar{m} m} g_{\bar{n} n} \sin \left(\gamma_{\bar{m}} x_{1}\right) \sin \left(\psi_{\bar{n}} x_{2}\right)
\end{aligned}
$$

where:

$$
f_{\bar{m} m}=\frac{4}{m \pi\left(1-\frac{\bar{m}^{2}}{m^{2}}\right)} ; \frac{\bar{m}}{m} \neq 1 \quad g_{\bar{n} n}=\frac{4}{n \pi\left(1-\frac{\bar{n}^{2}}{n^{2}}\right)} ; \frac{\bar{n}}{n} \neq 1 ; \quad \gamma_{\bar{m}}=\frac{\pi \bar{m}}{a} ; \psi_{\bar{n}}=\frac{\pi \bar{n}}{b}
$$

Introducing the above series expansions Eqs (11a-c) and (12) into Eqs (10a-e) will provide a unique solution to the all-edge clamped boundary condition.

Differentiating Eqs (10a-e) with respect to time and substituting in Eqs (5a-e) gives:

$$
\begin{aligned}
& C_{1}=-\sum_{m=1}^{\infty} \sum_{n=1}^{\infty} \sin \left(\alpha_{m} x_{1}\right) \sin \left(\beta_{n} x_{2}\right) e^{i \omega t}\left[\left(P_{1}+\frac{2 P_{2}}{R_{1}}\right) U_{m n}+\left(P_{2}+\frac{P_{3}}{R_{1}}\right) X_{m n}\right] \omega^{2} \\
& C_{2}=-\sum_{m=1}^{\infty} \sum_{n=1}^{\infty} \sin \left(\alpha_{m} x_{1}\right) \sin \left(\beta_{n} x_{2}\right) e^{i \omega t}\left[\left(P_{1}\right) V_{m n}+\left(P_{2}\right) Y_{m n}\right] \omega^{2} \\
& C_{3}=-\sum_{m=1}^{\infty} \sum_{n=1}^{\infty} \sin \left(\alpha_{m} x_{1}\right) \sin \left(\beta_{n} x_{2}\right) e^{i \omega t}\left[P_{1} W_{m n}\right] \omega^{2}
\end{aligned}
$$



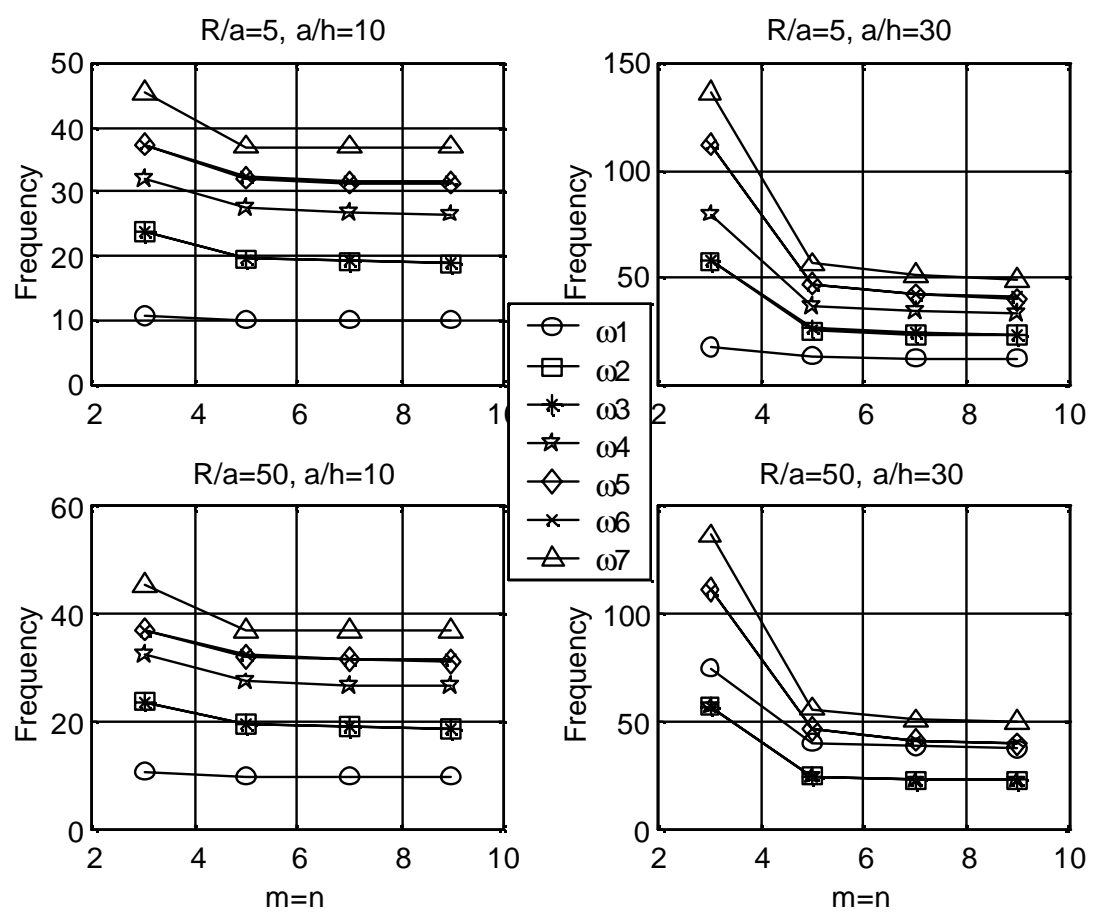

Fig. 3. Normalized natural frequencies for isotropic cylindrical panel with $b / a=2$.

$$
\begin{aligned}
C_{4} & =-\sum_{m=1}^{\infty} \sum_{n=1}^{\infty} \sin \left(\alpha_{m} x_{1}\right) \sin \left(\beta_{n} x_{2}\right) e^{i \omega t}\left[\left(P_{2}+\frac{P_{3}}{R_{1}}\right) U_{m n}+\left(P_{3}\right) X_{m n}\right] \omega^{2} \\
C_{5} & =-\sum_{m=1}^{\infty} \sum_{n=1}^{\infty} \sin \left(\alpha_{m} x_{1}\right) \sin \left(\beta_{n} x_{2}\right) e^{i \omega t}\left[\left(P_{2}\right) V_{m n}+\left(P_{3}\right) Y_{m n}\right] \omega^{2}
\end{aligned}
$$

A computer program is developed to solve for the eigenvalues (the frequencies of vibrations of the cylindrical shell) and the corresponding eigenvectors (the mode shapes of vibrations of the cylindrical shell).

\section{Finite element formulation}

Finite element analyses, using three different shell elements, were used for comparison with and validation of the analytical solutions. The three shell elements used are: (1) A four-node isoparametric element, developed by Bathe and Dvorkin [32], that uses reduced one-point integration approach as well as non-isoparametric form of shape functions for transverse shear deformations. This four-node shell element is implemented in NISA [33] finite element program. The element is denoted as NKPT32, NODRD = 1 in NISA; (2) A four-node quadrilateral shell element that is implemented in SAP2000 [34] finite element program; (3) An eight-node quadrilateral shell element (SHELL93) that is implemented in ANSYS [35] finite element program. All these shell elements have six degrees of freedom per each node (i.e., three translations along $x, y$ and $z$ coordinates and three rotations of the normal about $x, y$ and $z$ coordinates). The finite element analyses results are very comparable to the analytical solution results as will be shown in the subsequent section.

\section{Numerical results and discussion}

To illustrate the numerical results of the free vibration analysis of all edges clamped cylindrical panel with isotropic materials, cylindrical panels with square and rectangular plan projections are considered with the following values for modulus of elasticity, Possion's ratio and modulus of rigidity: 


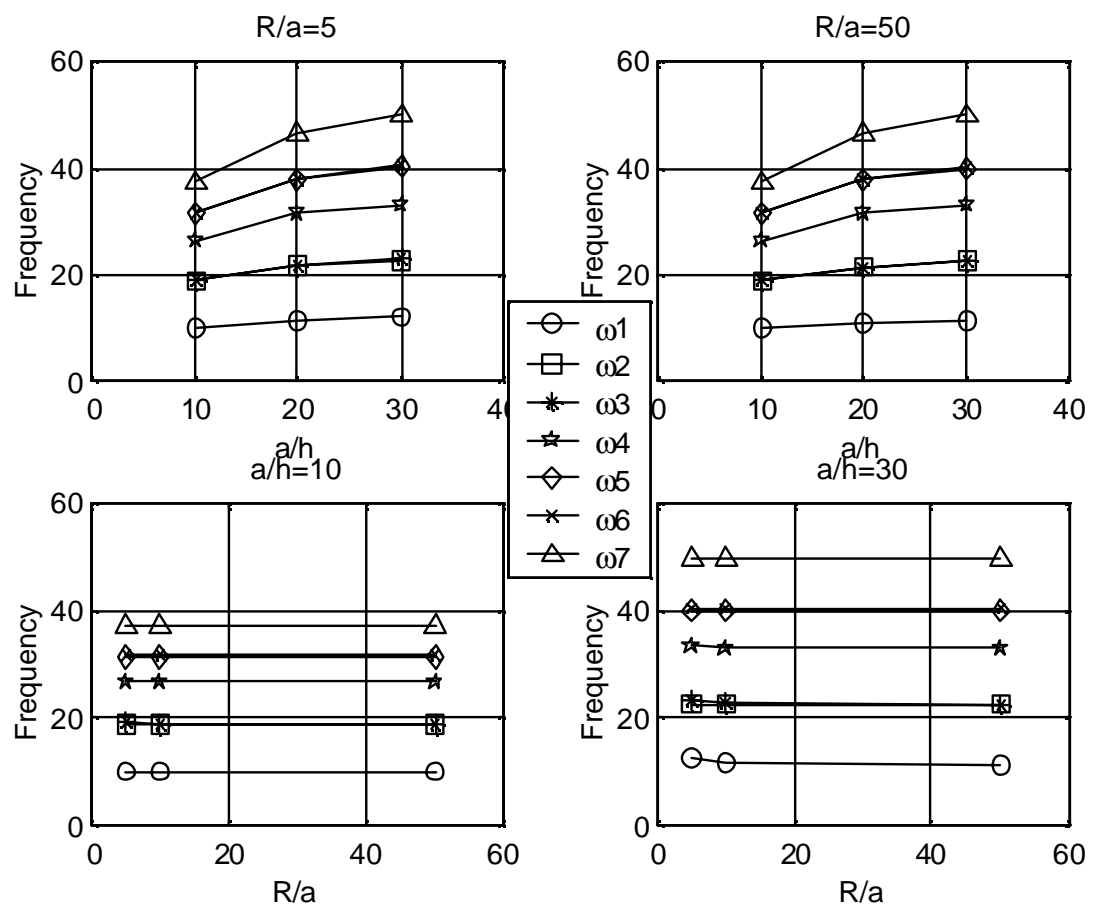

Fig. 4. Normalized natural frequencies for isotropic cylindrical panel $(b / a=1)$ with different $a / h$ and $R / a$.

$$
E_{1}=E_{2}=10000 \mathrm{MPa}, \nu_{12}=\nu_{21}=0.25 \text { and } G_{13}=G_{23}=G_{12}=4000 \mathrm{MPa}
$$

The eigenvalues are normalized for the sake of convenience in the following form:

$$
\omega_{\mathrm{Norm}}=\omega a^{2} \sqrt{\frac{\rho}{E_{2}}} / h
$$

Figure 2 shows the convergence of the analytical normalized natural frequencies $\left(\omega_{\text {Norm }}^{\prime} s\right)$ for square isotropic cylindrical panels with $b / a=1$ and various parametric values. It can be seen that the convergence of $\omega_{1}$ to $\omega_{7}$ is obtained at $m=n=5$. Figure 3 shows the convergence of the analytical normalized natural frequencies $\left(\omega_{\text {Norm }}^{\prime} s\right)$ for rectangular isotropic cylindrical panels with $b / a=2$ and various parametric values. It can be concluded that the convergence of $\omega_{1}$ to $\omega_{5}$ is obtained at $m=n=5$, while $\omega_{6}$ and $\omega_{7}$ converge at $m=n=7$. Figure 4 demonstrates that the frequency of vibration of square cylindrical panels increase with the increase in a/h ratio while the frequencies of these panels are not affected by the ratio of R/a. Figure 5(a) and (b) show sample mode shapes of the cylindrical panels with square plans with different parametric values. Figure 6(a) and (b) show selected mode shapes of the cylindrical panels with rectangular plans with different parametric values.

Tables 1 and 2 present a comparison between the results of the finite element solutions using NISA [33], SAP2000 [34] and ANSYS [35] - with $20 \times 20 \mathrm{mesh}$, and the analytical solutions for isotropic cylindrical panels with $b / a=1, R / a=10, a / h=10$ and $b / a=1, R / a=10, a / h=20$, respectively. Tables 3 and 4 present a comparison between the results of the finite element solutions using NISA [33], SAP2000 [34] and ANSYS [35] with $204 \times 40$ mesh and the analytical solutions for isotropic cylindrical panels with $b / a=2, R / a=10, a / h=10$ and $b / a=2, R / a=10, a / h=20$, respectively. Figure 7(a) through Fig. 7(d) show the finite element frequencies' ratios to that of the analytical solutions and their deviation from unity for square and rectangular panels, with different shell thicknesses.

It can be concluded from the above results that, in general, the deviation between the analytical solution and the finite element solution, however small, but it increases as the $b / a$ ratio increases and as the shell thickness decreases $(a / h$ ratio increases). It is also observed that the finite element solutions are normally underestimate the 
Table 1

Comparison of normalized frequencies between analytical and FEM solutions for panels with $b / a=1, R / a=10, a / h=10$

\begin{tabular}{crccc}
\hline Frequency & Analytical & FEM-NISA [33] & FEM-SAP [34] & FEM-ANSYS [35] \\
\hline$\omega_{1}$ & 9.897736 & 9.85779 & 9.8192 & 9.840725 \\
$\omega_{2}$ & 18.924930 & 18.99485 & 18.9490 & 18.940662 \\
$\omega_{3}$ & 18.947020 & 19.01682 & 18.9810 & 18.980246 \\
$\omega_{4}$ & 26.589800 & 26.65741 & 26.2690 & 26.761971 \\
$\omega_{5}$ & 31.330910 & 32.03936 & 31.0500 & 31.640865 \\
$\omega_{6}$ & 31.627800 & 32.34349 & 31.7320 & 31.922980 \\
$\omega_{7}$ & 37.179730 & 37.24426 & 36.7660 & 37.200855 \\
\hline
\end{tabular}
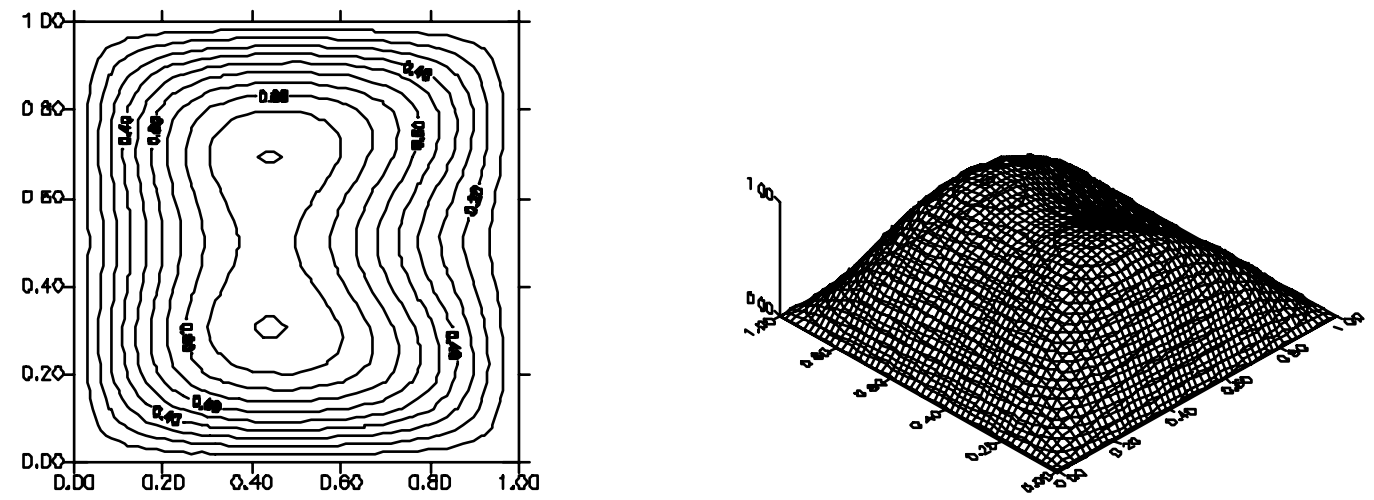

(a) Mode shape of U3(1,1) for an isotropic shell panel with $\mathrm{b} / \mathrm{a}=1, \mathrm{R} / \mathrm{a}=10$, $\mathrm{a} / \mathrm{h}=10$
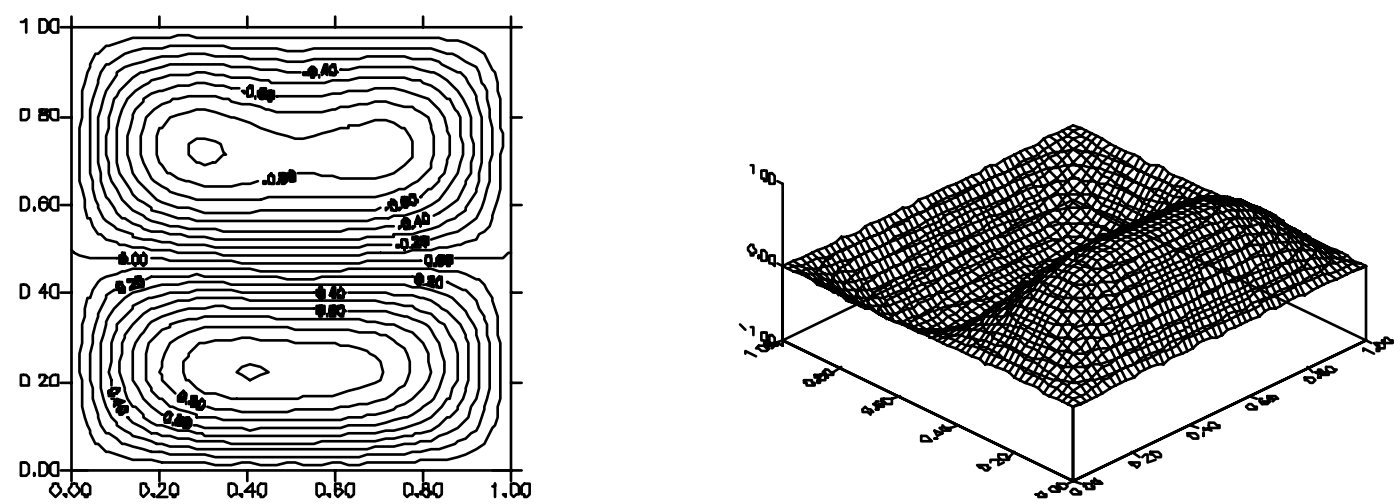

(b) Mode shape of $U 3(1,2)$ for an isotropic shell panel with $\mathrm{b} / \mathrm{a}=1, \mathrm{R} / \mathrm{a}=10$, $\mathrm{a} / \mathrm{h}=10$

Fig. 5. (a). Mode shape of U3(1,1) for an isotropic shell panel with $b / a=1, R / a=10, a / h=10$. (b). Mode shape of U3(1,2) for an isotropic shell panel with $b / a=1, R / a=10, a / h=10$.

natural frequencies for rectangular panels $(b / a=2)$ and for thin shells $(a / h=20)$ as shown in Fig. 7(d). These discrepancies may be due to the reduced integration schemes and/or exclusion of shell curvature in finite element formulations of shell elements.

It is also observed that the finite element analyses showed similar mode shapes to those obtained from the analytical solution and presented in Figs 5 and 6. 
Table 2

Comparison of normalized frequencies between analytical and FEM solution for panels with $b / a=1, R / a=10, a / h=20$

\begin{tabular}{ccccc}
\hline Frequency & Analytical & FEM-NISA [33] & FEM-SAP [34] & FEM-ANSYS [35] \\
\hline$\omega_{1}$ & 10.90719 & 10.66991 & 10.7156 & 10.492165 \\
$\omega_{2}$ & 21.60532 & 21.34325 & 21.4720 & 21.014742 \\
$\omega_{3}$ & 21.66954 & 21.40822 & 21.5440 & 21.063750 \\
$\omega_{4}$ & 31.32558 & 30.90899 & 30.9100 & 30.557643 \\
$\omega_{5}$ & 37.59598 & 38.13888 & 38.4400 & 36.843342 \\
$\omega_{6}$ & 37.84910 & 38.38602 & 38.6940 & 37.095926 \\
$\omega_{7}$ & 46.43315 & 46.67562 & 46.5060 & 45.603359 \\
\hline
\end{tabular}
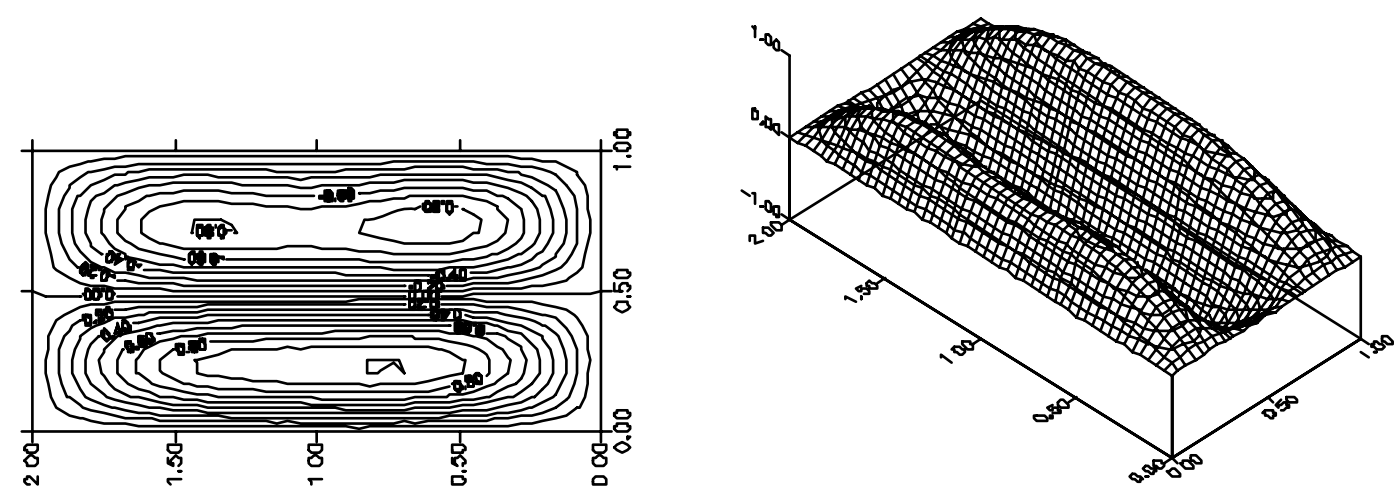

(a) Mode shape of U3(3,1) for an isotropic shell panel with $\mathrm{b} / \mathrm{a}=2, \mathrm{R} / \mathrm{a}=10, \mathrm{a} / \mathrm{h}=10$
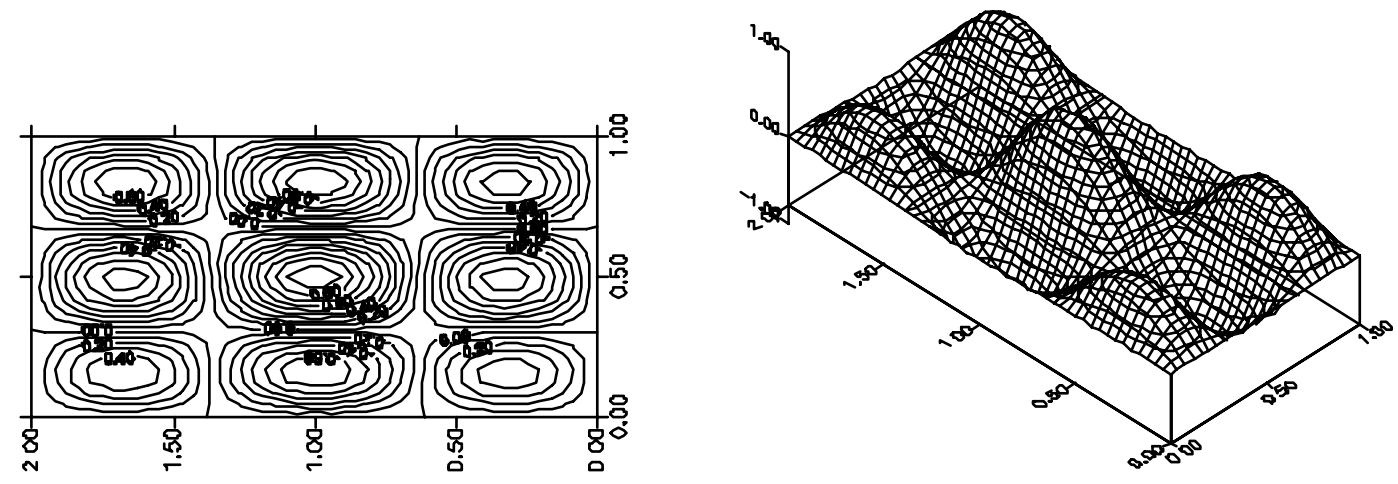

(b) Mode shape of U3(3,3) for an isotropic shell panel with $\mathrm{b} / \mathrm{a}=2, \mathrm{R} / \mathrm{a}=10$, a/h $=10$

Fig. 6. (a). Mode shape of U3(3,1) for an isotropic shell panel with $b / a=2, R / a=10, a / h=10$. (b). Mode shape of U3(3,3) for an isotropic shell panel with $b / a=2, R / a=10, a / h=10$.

\section{Conclusions and recommendations}

The free vibration response of shear-flexible cylindrical panels fabricated with isotropic materials, with all edges clamped boundary conditions was studied using a recently developed analytical solution approach and a well established four-node finite element model. The results of the analytical solution for various parametric ratios were compared with those of the finite element solution and the results were very comparable. 
Table 3

Comparison of normalized frequencies between analytical and FEM solution for panels with $b / a=2, R / a=10, a / h=10$

\begin{tabular}{crccc}
\hline Frequency & Analytical & FEM-NISA [33] & FEM-SAP [34] & FEM-ANSYS [35] \\
\hline$\omega_{1}$ & 6.966109 & 6.863061 & 6.9404 & 6.881345 \\
$\omega_{2}$ & 8.941903 & 8.766849 & 8.9229 & 8.813424 \\
$\omega_{3}$ & 12.351940 & 12.118350 & 12.3410 & 12.198804 \\
$\omega_{4}$ & 16.776780 & 16.768830 & 16.8940 & 16.793069 \\
$\omega_{5}$ & 17.176630 & 16.771000 & 17.0430 & 16.879777 \\
$\omega_{6}$ & 18.493520 & 18.403500 & 18.7200 & 18.497069 \\
$\omega_{7}$ & 21.362400 & 21.198580 & 21.6650 & 21.389219 \\
\hline
\end{tabular}

(a) $b / a=1, R / a=10, a / h=10$

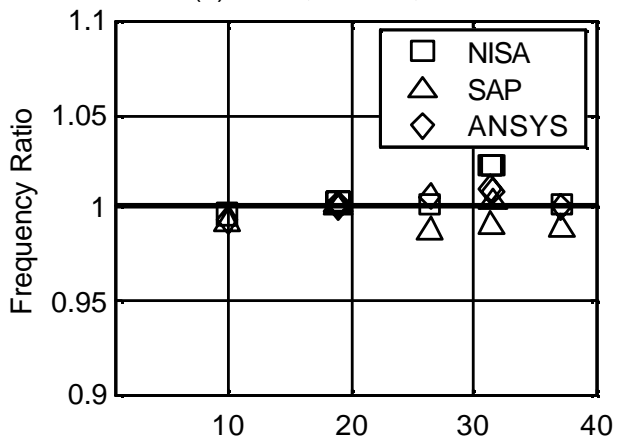

(c) $b / a=2, R / a=10, a / h=10$

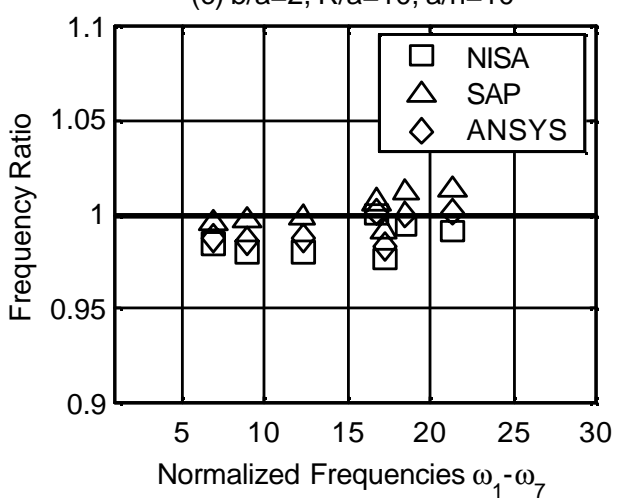

(b) $b / a=1, R / a=10, a / h=20$

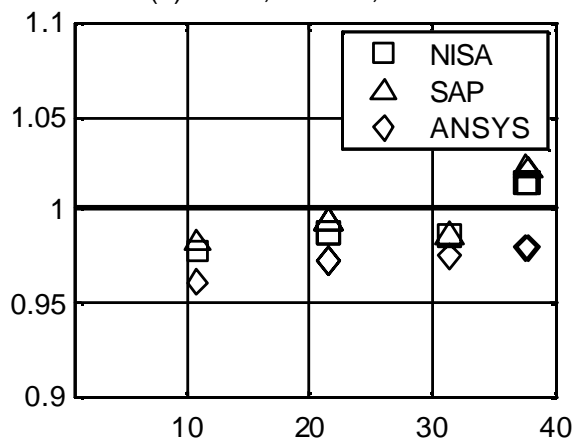

(d) $b / a=2, R / a=10, a / h=20$

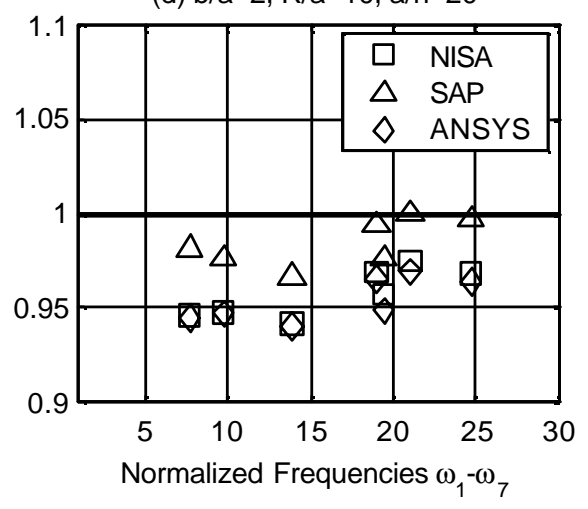

Fig. 7. a-d. Comparison of ratios of finite element solutions to analytical solutions.

The following conclusions are drawn from this study:

- The convergence rate of a cylindrical shell panel with square plan $(b / a=1)$ is faster than that of a cylindrical shell panel with rectangular plan with an aspect ratio $b / a=2$.

- The values of normalized natural frequencies increase with the increase of the ratio of segment length over the shell thickness, i.e., with the increase of $a / h$ ratio or decrease in shell thickness.

- The fundamental frequency (first mode) is not significantly affected by the ratio of radius of curvature for $R / a>$ 15 for $a / h=10$ to 30 as studied here. In higher frequency, the influence reduces further as R/a increases. This is due to the use of shallow shell theory of Sander's

- The deviations between the analytical solutions and the finite element solutions are more apparent as $b / a$ and $a / h$ increases.

- The analytical approach presented in this paper can be extended further to study the buckling, thermal and impact loading response of cylindrical panels. 
Table 4

Comparison of normalized frequencies between analytical and FEM solution for panels with $b / a=2, R / a=10, a / h=20$

\begin{tabular}{crccc}
\hline Frequency & Analytical & FEM-NISA [33] & FEM-SAP‘[34] & FEM-ANSYS [35] \\
\hline$\omega_{1}$ & 7.645855 & 7.233375 & 7.5106 & 7.225412 \\
$\omega_{2}$ & 9.842625 & 9.318339 & 9.6092 & 9.317587 \\
$\omega_{3}$ & 13.866130 & 13.052530 & 13.3990 & 13.035096 \\
$\omega_{4}$ & 18.982900 & 18.397500 & 18.8700 & 18.314229 \\
$\omega_{5}$ & 19.480500 & 18.634570 & 19.0328 & 18.467538 \\
$\omega_{6}$ & 21.091580 & 20.572960 & 21.0980 & 20.445485 \\
$\omega_{7}$ & 24.712600 & 23.920320 & 24.6480 & 23.834635 \\
\hline
\end{tabular}

\section{References}

[1] A.E.H. Love, Treatise on the Mathematical Theory of Elasticity, Dover, New York, 1944.

[2] L.H. Donnell, A discussion of thin Shell Theory, Proceedings of the 5th International Congress of Applied Mechanics, 1938.

[3] J.L. Sanders, An Improved First-Approximation Theory for Thin Shells, NASA Tech. Report, NASA-TRR24, 1959.

[4] S. Timoshenko and S. Woinowsky-Krieger, Theory of Plates and Shells, (2nd Edition), McGraw Hill, Singapore, 1985.

[5] W. Flugge, Stresses in Shells, (2nd Edition,), Springer Verlag, 1990.

[6] A.W. Leissa, Vibration of shells, NASA SP-288, 1973.

[7] A.K. Noor, Bibliography of monographs and surveys on shells, ASME Applied Mechanics Review 43(9) (1990), $223-234$.

[8] K.M. Liew, C.W. Lim and S. Kitipornchai, Various theories for vibration of shallow shells: a review with bibliography, ASME Applied Mechanics Reviews 50(8) (1997), 431-44.

[9] M.S. Qatu, Recent research advances in the dynamic behavior of shells: part 2. Homogeneous shells, Applied Mechanics Reviews 55(5) (2002), 415-434.

[10] W. Soedel, Vibrations of Shells and Plates, (Third Edition), Marcel Dekker, Inc. 2004.

[11] H.T. Yang, S. Saigal, A. Masud and R. Kapania, A survey of recent shell finite elements, International Journal of Numercial Methods in Engineering 47(1-3) (2000), 101-127.

[12] C. Wang and J.C.S. Lai, Prediction of natural frequencies of finite length circular cylindrical shells, Applied Accoustics 59 (2000), 385-400.

[13] J. Callahan and H. Baruh, A closed-form solution procedure for circular cylindrical shell vibrations, International Journal of Solids and Structures 36 (1999), 2973-3013.

[14] Y. Xiang, Y.F. Ma, S. Kitipornchai, C.W. Lim and C.W.H. Lau, Exact solutions for vibration of cylindrical shells with intermediate ring supports, International Journal of Mechanical Sciences 44 (2002), 1907-1924.

[15] A.J. Stanley and N. Ganesan, Free vibration characteristics of stiffened cylindrical shells, Computers \& Structures 65(1) (1997), 33-45.

[16] W.Q. Chen and H.J. Ding, Natural frequencies of fluid-filled transversely isotropic cylindrical shells, International Journal of Mechanical Sciences 41 (1999), 677-684.

[17] W.Q. Chen, H.J. Ding and R.Q. Xu, On exact analysis of free vibrations of embedded transversely isotropic cylindrical shells, International Journal of Pressure Vessels and Piping 75 (1998), 961-966.

[18] T.Y. Ng, K.Y. Lam and J.N. Reddy, Dynamic stability of cylindrical panels with transverse shear effects, International Journal of Solids and Structures 36 (1999), 3483-3496.

[19] L.V. Kurpa, K.I. Lyubitska and A.V. Shmatko, Solution of vibration problems for shallow shells of arbitrary form by the R-function method, Journal of Sound and Vibration 279 (2005), 1071-1084.

[20] E.L. Jansen, A comparison of analytical - numerical models for nonlinear vibrations of cylindrical shells, Computers and Structures $\mathbf{8 2}$ (2004), 2647-2658.

[21] M. Amabili, Nonlinear vibrations of circular cylindrical panels, Journal of Sound and Vibration 281 (2005) $509-535$.

[22] C.W. Bert and M. Kumar, Vibration of cylindrical shells of bimodulus composite materials, Journal of Sound and Vibration 81 (1982), $107-121$.

[23] J.N. Reddy, Exact solutions of moderately thick laminated shells, Journal of Engineering Mechanics 110(5) (1984), 794-809.

[24] M.E. Vanderpool and C.W. Bert, Vibration of a materially monoclinic, Thick-Wall Circular Cylindrical Shell AIAA Journal 19(5) (1981), 634-641.

[25] K.M. Liew, C.W. Lim and S. Kitipornchai, Vibration characteristics of cantilevered thick cylindrical shallow shells, AIAA Journal 34(11) (1996), 2451-2453.

[26] R.A. Chaudhuri and H.R.H. Kabir, A boundary-continuous-displacement based Fourier analysis of laminated doubly curved panels using classical shallow shell theories, International Journal of Engineering Science 30 (1992), 1647-1664.

[27] H.R.H. Kabir and R.A. Chaudhuri, A direct Fourier approach for the analysis of thin finite-dimensional cylindrical panels, Computers and Structures 46(2) (1993), 279-287.

[28] H.R.H. Kabir, Boundary value problems of thin, moderately deep cross-ply laminated cylindrical shells, Composite Structures $\mathbf{1 8}$ (1991), 297-310.

[29] R.A. Chaudhuri and H.R.H. Kabir, Fourier solution to HOT-Based laminated Shell-Boundary value problem, International Journal of American Institute of Aeronautics and Astronautics (1995), 1681-1688.

[30] R.A. Chaudhuri and H.R.H. Kabir, Effect of boundary constraint on the frequency response of moderately thick doubly curved cross-ply panels using mixed Fourier solution functions, Journal of Sound and Vibration 283 (2005), 263-293.

[31] A.E. Green, Double Fourier series and boundary value problems, Proceedings of Cambridge Philosophical Society 40 (1944), $222-228$. 
[32] K.J. Bathe and E.N. Dvorkin, A four-node plate bending element based on Mindlin-Reissner plate theory and mixed interpolation, International Journal of Numerical Methods 21 (1985), 367-383.

[33] NISA-II, Engineering Mechanics Research Corporation, Troy, MI, USA, 1990.

[34] SAP2000. Computers and Structures Inc., 1995 University Ave., Berkeley, Ca, 94720, USA. www.csiberkeley.com.

[35] ANSYS. ANSYS, Inc., 275 Technology Drive, Canonsburg, PA 15317, www.ansys.com. 

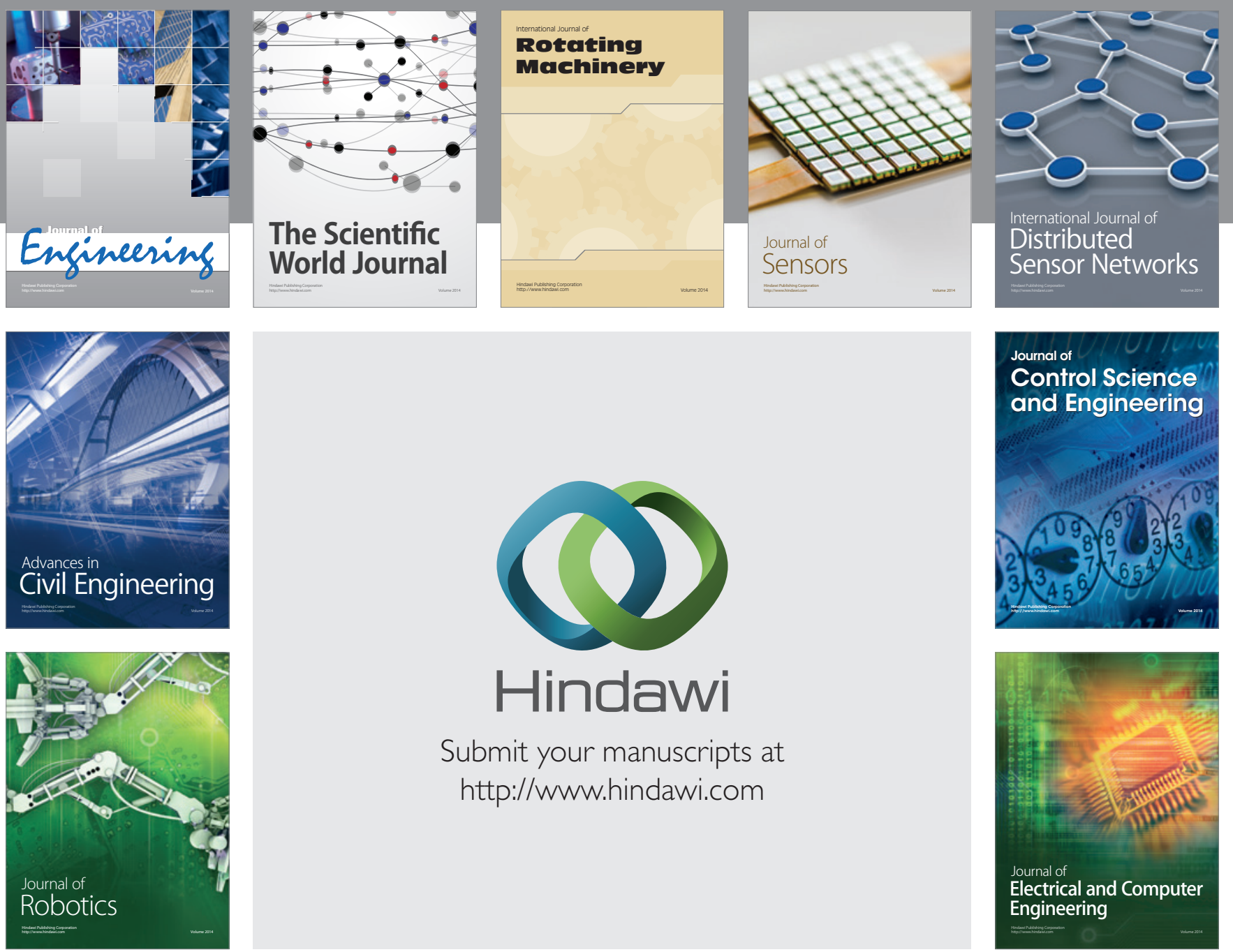

Submit your manuscripts at

http://www.hindawi.com
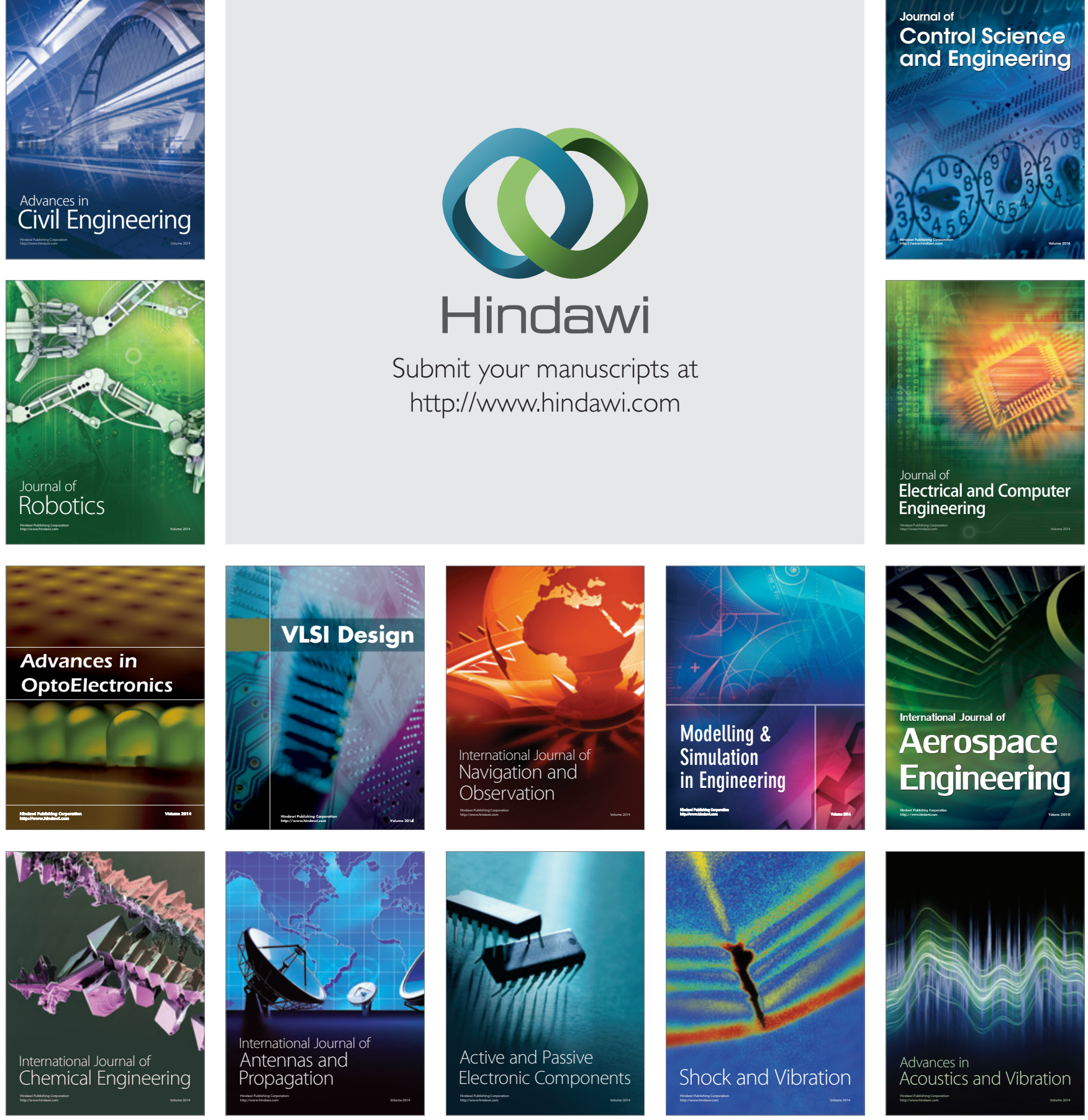